\title{
Surgical Management and Angioembolization in a Hybrid Operating Room for a Patient with a Pelvic Bone Fracture and Other Lethal Injuries
}

\author{
Pil Young Jung, Keum Seok Bae \\ Department of Surgery, Yonsei University Wonju College of Medicine, Wonju Severance Christian Hospital, Trauma center
}

The current management of pelvic fracture patients with other lethal injuries who are hemodynamically unstable consists of aggressive resuscitation, emergency operation, and angioembolization. Despite this multidisciplinary approach, a high mortality rate was seen in these high-risk patients with pelvic bone fracture and other lethal injuries. Therefore, we pursued proper therapies to improve patient outcomes. We have experienced successful treatment for patients with pelvic bone plus other lethal injuries.

(Trauma Image Proced 2017(1):27-28)

Key Words: Pelvic Bones; Fractures, Bone; Surgical Procedures, Operative; Endovascular Procedures

\section{CASE}

A 14-year-old female patient with no medical history was admitted via the emergency room with blunt trauma. At admission, she was in shock status and had a positive focused abdominal sonography for trauma (FAST) sign with a right acetabulum wall fracture, superior and inferior ramus fractures, and an open book type fracture of the pelvis (Fig. 1). In addition, she had a hemoperitoneum and peritonitis due to bowel perforation and a mesenteric injury. We planned to perform an emergency operation in a hybrid operating room with angioembolization at the same time. First, segmental resection of the jejunum was done and then angioembolization of internal iliac artery branch was performed (Fig. 2., 3.). Finally, external fixation of the

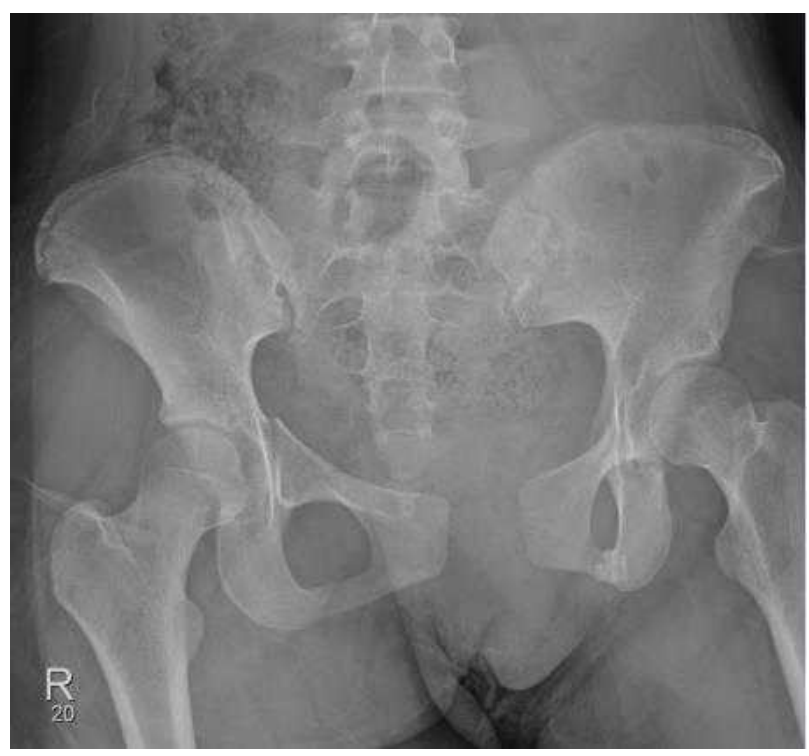

Fig. 1. Pelvic anteroposterior view radiograph shows an unstable pelvic bone fracture.

Received: February 7, 2017 Revised: March 1, 2017 Accepted: March 5, 2017

Correspondence to: Keum Seok Bae, Department of Surgery, Yonsei University Wonju College of Medicine, Wonju Severance Christian Hospital, 65, 220-701, 20 IIsan-ro, Wonju-si, Gangwon-do, Korea

Tel: 82-33-741-0881, Fax: 82-33-741-1205, E-mail: bksgs@yonsei.ac.kr

Copyright (c) 2017 Korean Association for Research, Procedures and Education on Trauma. All rights reserved.

(c) This is an open-access article distributed under the terms of the Creative Commons Attribution Non-Commercial License (http://creativecommons.org/ licenses/by-nc/4.0) which permits unrestricted noncommercial use, distribution, and reproduction in any medium, provided the original work is properly cited 


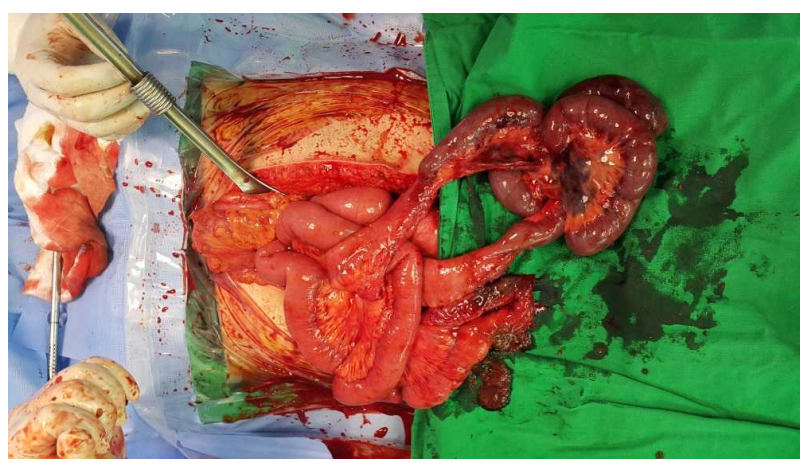

Fig. 2. Operative finding is severe ileal and accompanied mesenteric injury.

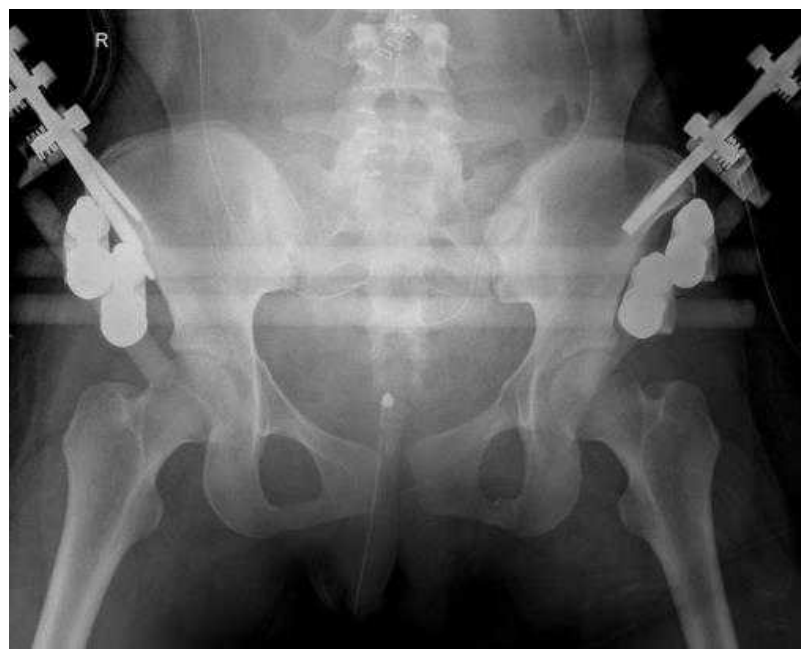

Fig. 4. Radiograph shows external fixation.

pelvis was done (Fig. 4.). After 7 days, internal fixation of the pelvis was done. The patient recovered and underwent rehabilitation without complications.

\section{DISCUSSION}

Major pelvic disruption with hemorrhage has a high rate of mortality and pelvic fracture hemorrhage remains a management challenge (1). Earlier intervention by trauma surgeons with techniques, such as preperitoneal packing, aortic balloon occlusion, and use of hybrid operative suites, may improve survival outcomes (2).

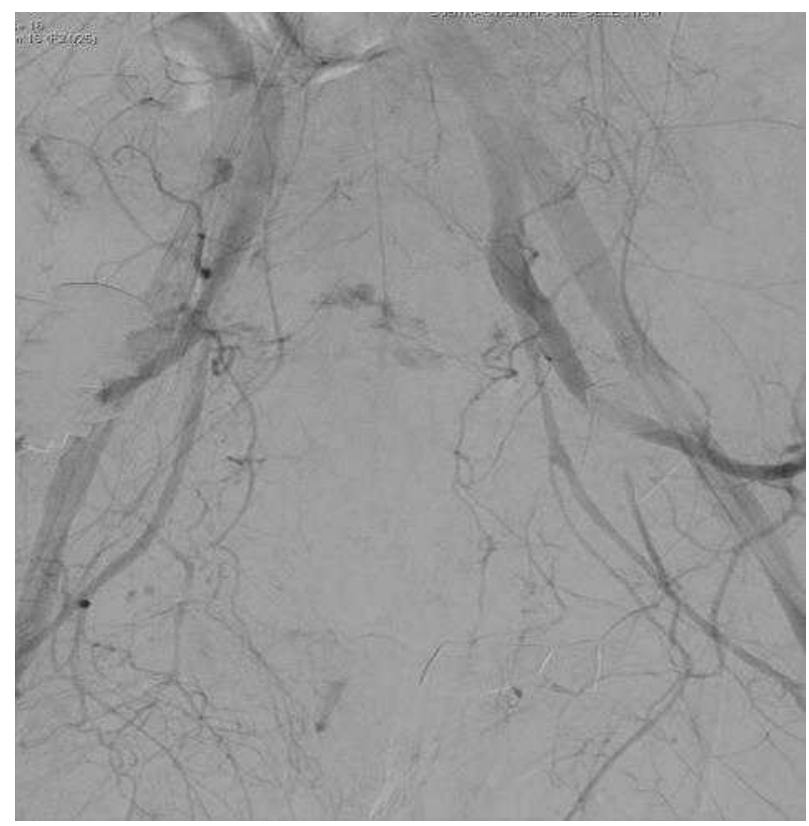

Fig. 3. Iliac arteriogram shows contrast extravasation from a branchs of the internal iliac artery.

Hybrid operation room is useful for the multiple-trauma patient who needs simultaneous operation and angioembolization without any time delay.

\section{Conflict of Interest Statement}

No potential conflict of interest relevant to this article was reported.

\section{REFFERENCES}

1. Burlew CC, Moore EE, Smith WR, Johnson JL, Biffl WL, Barnett CC, et al. Preperitoneal pelvic packing/external fixation with secondary angioembolization: optimal care for life-threatening hemorrhage from unstable pelvic fractures. Journal of the American College of Surgeons. 2011;212(4): 628-35.

2. Tesoriero RB, Bruns BR, Narayan M, Dubose J, Guliani SS, Brenner ML, et al. Angiographic embolization for hemorrhage following pelvic fracture: Is it "time" for a paradigm shift? Journal of Trauma and Acute Care Surgery. 2017;82(1):18-26. 